INNOVATIONS IN PRIMARY CARE

\title{
EMR Happy Hour: New Approach to Electronic Medical Record Continuous Learning
}

\author{
Margaret A. Day, MD, MSPH \\ Jeffery L. Belden, MD \\ University of Missouri, Columbia, Missouri \\ Ann Fam Med 2019;17:373. https://doi.org/10.1370/afm.2394.
}

\section{THE INNOVATION}

We developed an innovative approach to peer-to-peer sharing of best practices using the Electronic Medical Record (EMR). Why? The challenges of EMR training are twofold: (1) even the most easily adopted EMR innovations and features must be communicated effectively to existing users, and (2) time pressures result in an inertia with regard to workflows and habits of existing users. Our goals include empowering clinicians to achieve skills in chart review, ordering, and documentation to improve self-efficacy and timeliness.

\section{WHO AND WHERE}

Family Medicine at MU Health Care, a 60-faculty academic department using a Cerner EMR.

\section{HOW}

We engage with our family medicine faculty peers during hour-long monthly "EMR Happy Hours." Topics are chosen deliberately and focus on high-yield, low-effort interventions. We devote many sessions to reducing documentation burden, showing a variety of documentation options to help people find the most efficient method that suits their style. We introduced a Google Doc for sharing users' favorite commonly used text expansion phrases (auto-texts) which they can copy or modify, ranging from short phrases to entire note templates (very popular and big time-savers). We designed, built, and demonstrated an efficient, new, single page of ready-to-use common clinic orders. Other topics are included in supplemental materials.

Each participant, usually 3 to 4 in attendance per session, but sometimes 8 to 10 , brings a laptop to allow real-time practice of new skills. Facilitators for the sessions include a member of the EMR training staff as well as 1 to 3 clinical faculty mem-

Conflicts of interest: authors report none.

\section{Corresponding author}

Margaret A. Day, MD, MSPH

305 N Keene Street, Suite 203

Columbia, MO 65201

daym@health.missouri.edu bers, aiming for a small 1:3 to 1:4 teacher-to-student ratio. This could be replicated in other practice settings with a clinician willing to share best EMR practices during regularly scheduled meeting times, early mornings, or lunch times.

When attendees describe difficulties with an aspect of EMR use, we can witness their actions, allowing us to explain, coach at the elbow, reconfigure their preferences, or diagnose a system problem. We report systems issues to the help desk, relieving the clinician attendees of reporting burden.

We start with a flexible plan, allowing users to dictate the agenda based on their experiences and needs. We encourage participants to share one item learned with a colleague not able to attend the session.

\section{LEARNING}

We expected EMR Happy Hour to help physicians save time and lighten documentation burden. An unexpected outcome is that all participants, clinician and EMR trainer facilitators included, learn something new during the sessions. Surveying participants, we learned they valued peer support in particular, feeling less isolated while learning alongside others who share their plight. One physician responded, "Collegial. Get to talk with others in the same situation as me." Another states, "Excellent opportunity to learn new EMR tricks, work-arounds, strategies to make life easier. Good place to share EMR frustrations and see if others have a different system." The unique aspect of peers training peers during "EMR Happy Hour" allows for innovative, trusted, high-yield learning which often does improve individual physician efficacy/efficiency in the EMR. Attendees include our department's most productive clinicians (2.9 to 3.3 visits per hour). Perhaps the most powerful reason busy physicians consistently attend is the intentional monthly opportunity to acknowledge the inefficiencies are often in the tool or the system or the workflows; not the provider. That acknowledgment is a powerful medium to achieve self-efficacy and resist the forces contributing to physician burnout related to their work environment.

To read or post commentaries in response to this article, see it online at http://www.AnnFamMed.org/content/17/4/373.

Submitted January 15, 2019; submitted, revised, February 8, 2019; accepted February 27, 2019.

Key words: health information technology; electronic medical record; burnout

Acknowledgment: Robert P. Pierce, MD, MSPH, and Aaron D. Gray, MD.

Supplementary materials: Available at http://www. AnnFamMed. org/content/17/4/373/suppl/DC1/. 